\title{
In Silico Determination of Binding Free Energy and Bonding Interactions between Monosubstituted Cefovecin and Pseudomonas
}

\author{
Aeruginosa Lipase \\ O.V.Ikpeazu' ${ }^{1}$ K.K. Igwe'2 I. E. Otuokere ${ }^{3}$ \\ ${ }^{1}$ Dept of Biochemistry, Abia State University, Uturu,Nigeria \\ ${ }^{2}$ Dept of Vet Biochemistry and Pharmacology, Michael Okpara University of Agriculture, Umudike, Nigeria. \\ ${ }^{3}$ Dept of Chemistry, Michael Okpara University of Agriculture, Umudike, Nigeria
}

\begin{abstract}
Z)- 2-(2-amino- 1,3-thiazol- 4-yl)- 2methoxyiminoacetyl] amino)-8-oxo-3[(2S)-oxolan-2-yl]-5thia-1-azabicyclo[4.2.0]oct-2-ene-2-carboxylic acid (cefovecin) is an antibiotic of the cephalosporin class. In silico binding free energy of six analogous structurally diverse cefovecin with Pseudomonas aeruginosa lipase were determined using Patchdock and Firedock softwares. The bonding interactions were also studied. The binding energy of cefovecin was $-44.30 \mathrm{kcal} / \mathrm{mol}$. The free binding energies of $\mathrm{COOH}, \mathrm{COCH}_{3}, \mathrm{CH}_{3}, \mathrm{NO}_{2}, \mathrm{CF}_{3}$ and $\mathrm{NH}_{2}$ analogueswere $-44.30,-43.55,-39.49,-43.40,-30.25$ and $-44.18 \mathrm{Kcal} / \mathrm{mol}$ respectively. All the monosubstituted analogues showed lower negative values than the non substituted cefovecin. These lower negative values indicate that that the reactions are feasible. Their inhibition is lower compared to cefovecin against Pseudomonas aeruginosa lipase. The modes of bonding of six analogous structurally diverse cefovecin with Pseudomonas aeruginosa lipase were attributed to hydrogen bonding and steric interaction.
\end{abstract}

Keywords- Binding energy, cefovecin, docking, Pseudomonas aeruginosa lipase, scoring function

\section{INTRODUCTION}

Cefovecin is an antibiotic of the cephalosporin class, licensed for the treatment of skin infections in cat and dogs. It is the first single-dose injectable antibiotic for dogs and cats that assures owner compliance with dosing for the animal. It is used for the treatment of skin infections caused by Pasturella multocida in cats, and Staphylococcus intermedius/S. canis in dogs. The advantage of using injectable antibiotics is not missing a dose that can allow partially resistant microbes to recover during missed doses. The disadvantage is the durable presence of subtherapeutic concentrations in the weeks after the resolution of infections. This is associated with the development of resistance in microbes. It is effective, but should not be used in pregnant or lactating animals or in animals with a history of allergies to penicillin or cephalosporin drugs [1]. Cefovecin was first authorized for use in the European Union in June 2006 [2], and was approved for use in the United States in June 2008 [3]. It is approved as a broad-spectrum, third-generation cephalosporin for subcutaneous injection lasting 14 days for treating skin and soft tissue infections [4]. Contraindications include known allergies to cefovecin or antibiotics containing $\beta$-lactam rings such as penicillin or cephalosporins. Adverse reactions can include anaphylaxis. It is not for use in humans and should be kept out of reach of children. Individuals with similar known allergies should avoid dermal contact when handling the drugs. Adverse reactions in dogs can include lethargy, decreased appetite, vomiting, diarrhea, blood in feces, and flatulence. Adverse reactions in cats can include vomiting, diarrhea, decreased appetite, lethargy, odd hyperactive behavior, and inappropriate urination. Mildly increased serum ALT and gamma glutamyl transferase have been noted [5]. The following adverse events were reported voluntarily during post-approval use of the product in dogs and cats: death, tremors/ataxia, seizures, anaphylaxis, acute pulmonary edema, facial edema, injection site reactions (alopecia, scabs, necrosis, and erythema), hemolytic anemia, salivation, pruritis, lethargy, vomiting, diarrhea, and inappetance [6]. Cefovecin functions by interfering with cell wall synthesis. The chemical covalently binds to the so-called pencillin binding proteins. Due to the high protein-binding of the chemical, it is not effective against Pseudomonas spp., or enterococci. In drug studies, cefovecin administered to dogs 
was $92.4 \%$ effective against skin infections (secondary superficial pyoderma,abscesses, and infected wounds). In cats, it was $96.8 \%$ effective against skin infections [7]. Figure 1 shows the structure of cefovecin.

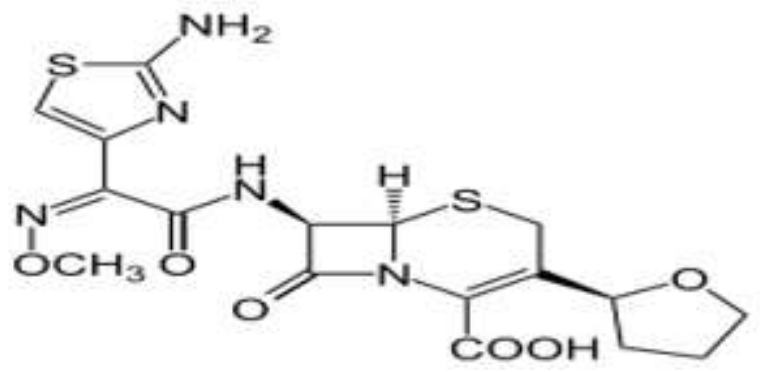

Fig.1: Structure of -7-([(2Z)- 2-(2-amino-1,3thiazol-4-yl)-2-methoxyiminoacetyl Jamino)-8-oxo3-[(2S)-oxolan-2-yl]-5-thia-1-azabicyclo[4.2.0]oct2-ene-2-carboxylic acid[ (cefovecin)

\section{METHODOLOGY}

\section{Protein preparation}

The crystal structure of Pseudomonas aeruginosa lipase was obtained from the Protein Data Bank, PDB 1ex9 (Figure 2). The protein structure was subjected to a refinement protocol using Molegro Molecular Viewer [8].

\section{Designing of structural analogs of Cefovecin}

The structure of cefovecin (Figure 1) was drawn with ACD/ChemSketch software [9]. The structural analogues of cefovecin were developed with structuralmodifications with different substituents. The carboxylic group [COOH] group at of cefovecin was replaced with $\mathrm{COCH}_{3}, \mathrm{CH}_{3}, \mathrm{NO}_{2}, \mathrm{CF}_{3}$ and $\mathrm{NH}_{2}$ analogues. The structures were built with ACD/ChemSketch software and minimized with Arguslab software [10].

\section{Molecular docking}

Molecular docking was performed using Patchdock [11]. Patchdock is a molecular docking algorithm based on shape complementarity principles. Refinement was done in Firedock [12, 13] and processed with Molegro molecular viewer [14]. Lipinski rule of 5 was evaluated using Sanjeevini: a freely accessible web-server for target directed lead molecule discovery [15].

\section{RESULTS AND DISCUSSION}

The Table 1: Estimated free energy of binding (FEB) of cefovecin and its analogues against Pseudomonas aeruginosa lipase is shown in Table 1. Crystal structure of Pseudomonas aeruginosa lipase PDB lex9 is shown in Figure 2. Cefovecin analogues in complex with
Pseudomonas aeruginosa lipase are presented in Figures 3 8. The bonding interactions are illusstrated in Figures 9-14.

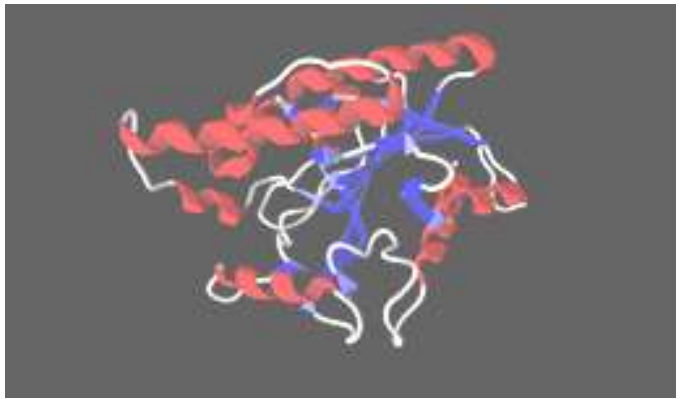

Fig.2: Crystal structure of Pseudomonas aeruginosa lipase $P D B$ lex 9

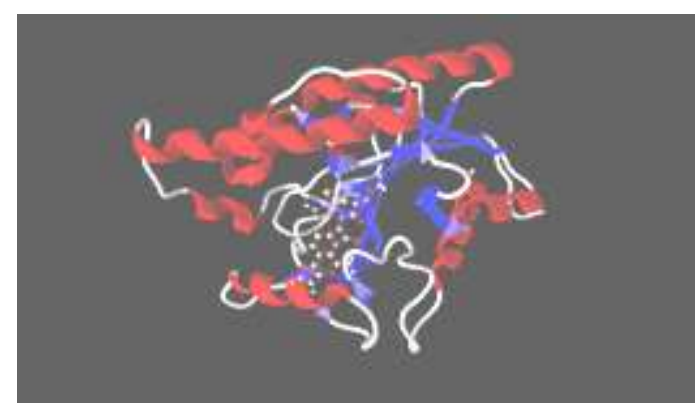

Fig.3: Cefovecin in complex with Pseudomonas aeruginosa lipase

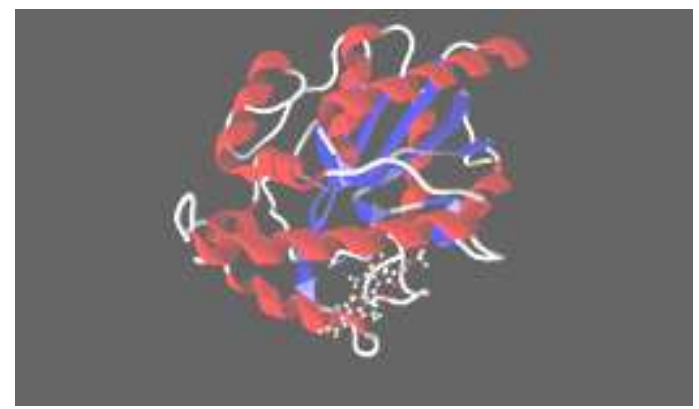

Fig.4: Cefovecin $\mathrm{COCH}_{3}$ analogue in complex with Pseudomonas aeruginosa lipase

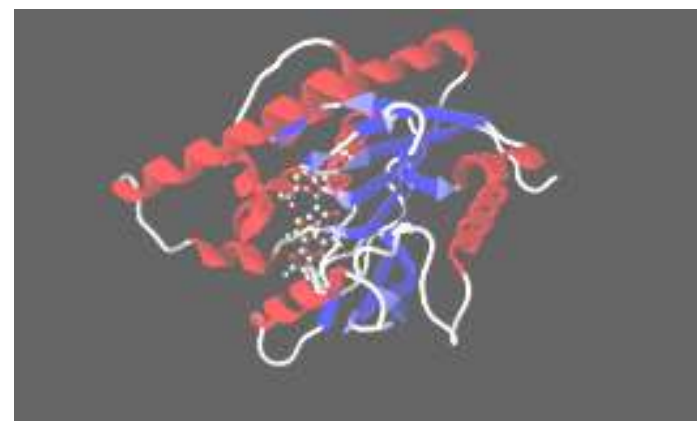

Fig.5: Cefovecin $\mathrm{CH}_{3}$ analogue in complex with Pseudomonas aeruginosa lipase 


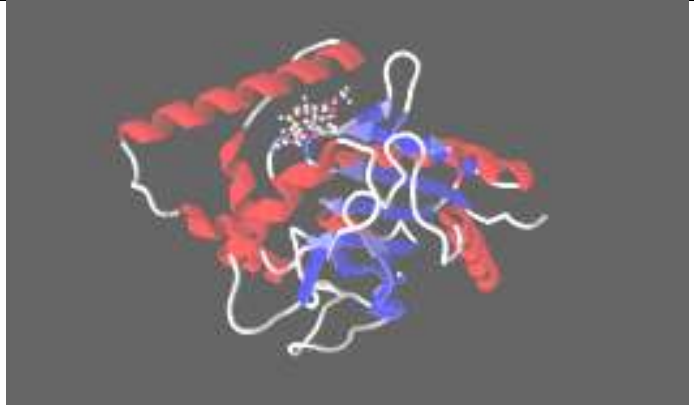

Fig.6: Cefovecin $\mathrm{NO}_{2}$ analogue in complex with Pseudomonas aeruginosa lipase

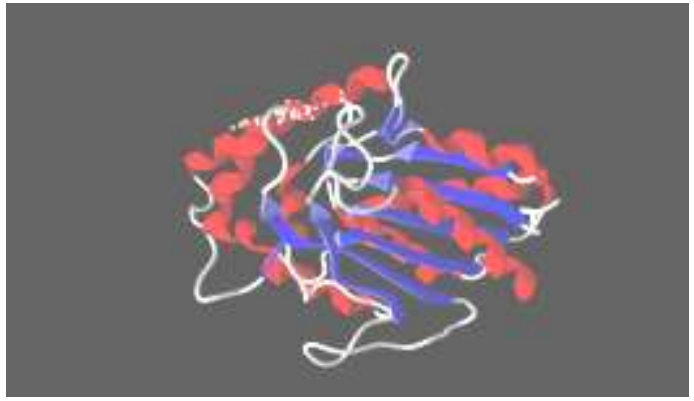

Fig.7: Cefovecin $\mathrm{CF}_{3}$ analogue in complex with Pseudomonas aeruginosa lipase

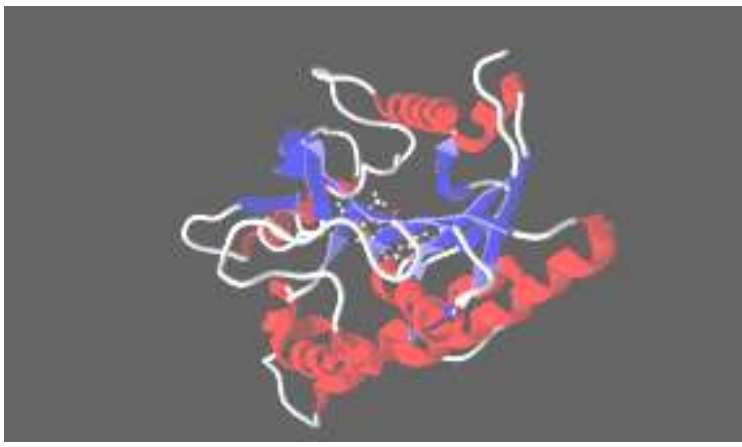

Fig.8: Cefovecin $\mathrm{NH}_{2}$ analogue in complex with Pseudomonas aeruginosa lipase

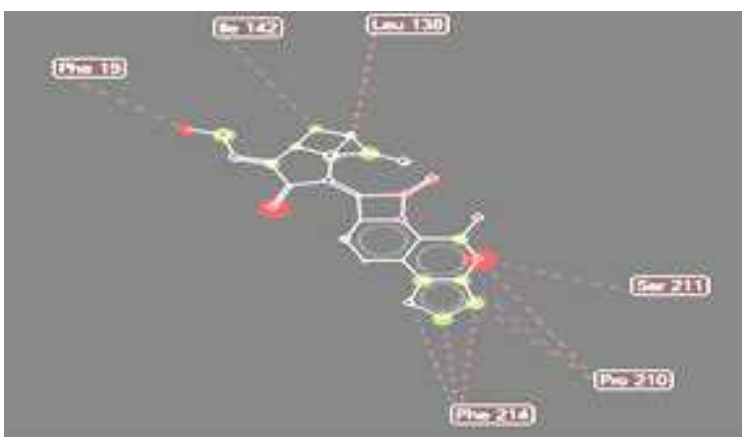

Fig.9: Steric interaction of Cefovecin with Pseudomonas aeruginosa lipase

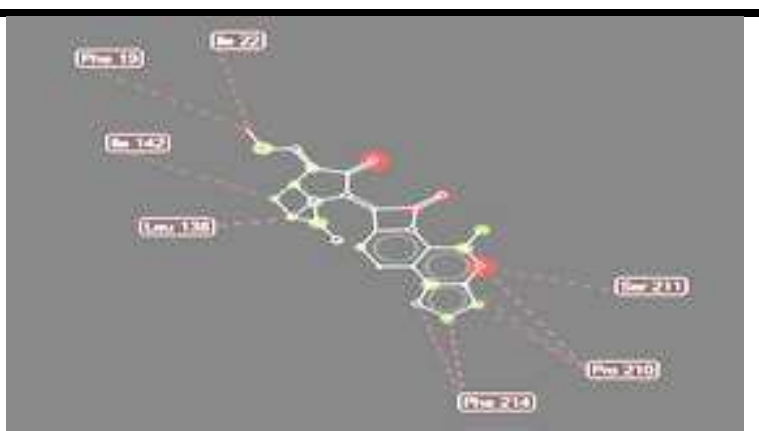

Fig.10: Steric interaction of Cefovecin $\mathrm{COCH}_{3}$ analogue with Pseudomonas aeruginosa lipase

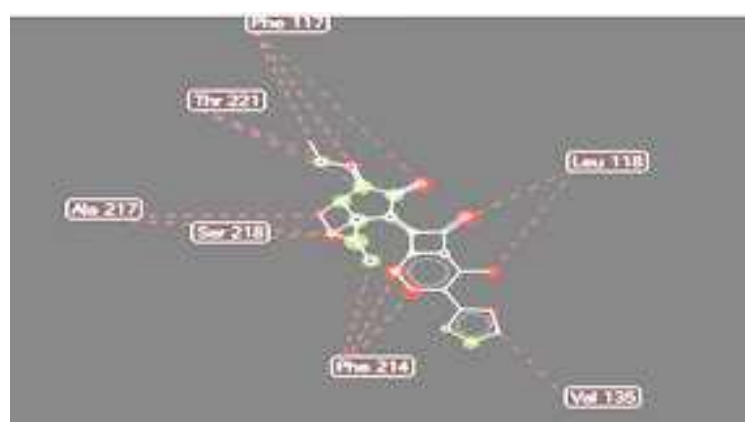

Fig.11: Steric interaction of Cefovecin $\mathrm{CH}_{3}$ analogue with Pseudomonas aeruginosa lipase

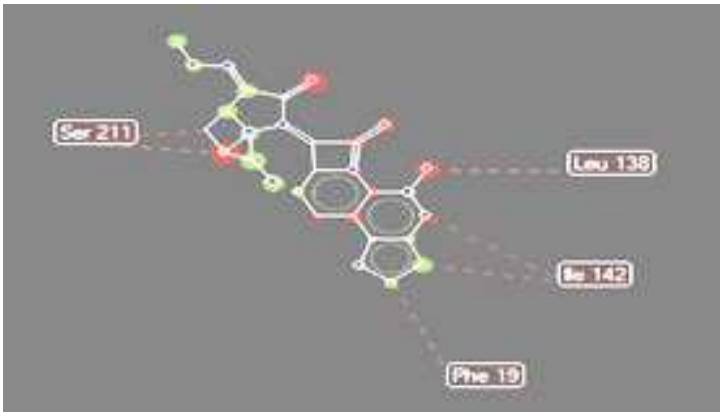

Fig.12: Steric interaction of Cefovecin $\mathrm{NO}_{2}$ analogue with Pseudomonas aeruginosa lipase

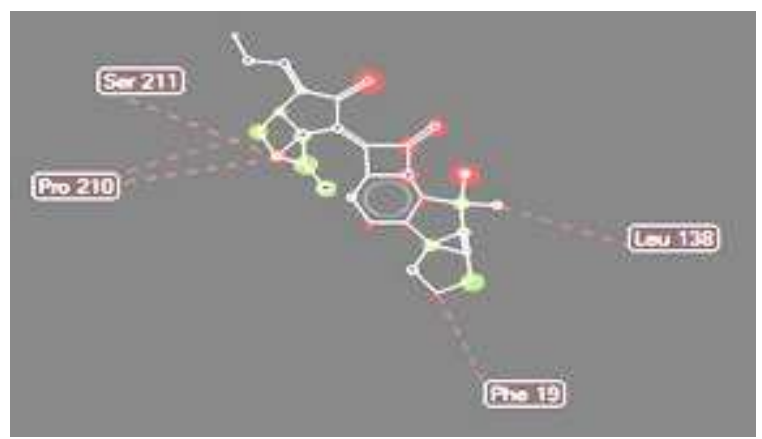

Fig.13: Steric interaction of Cefovecin $\mathrm{CF}_{3}$ analogue with Pseudomonas aeruginosa lipase 


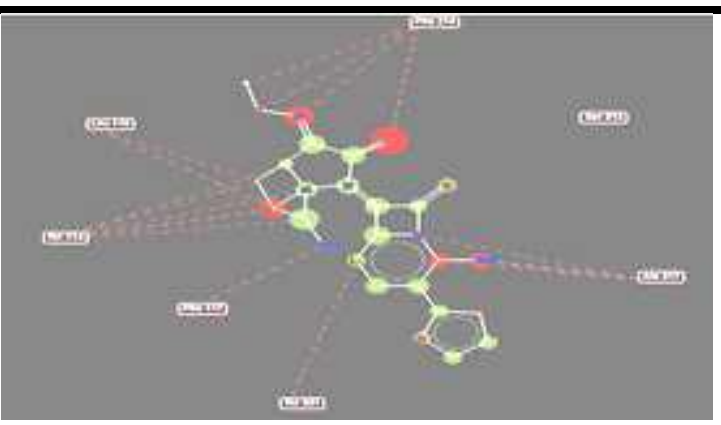

Fig 14: Hydrogen and steric interactions of cefovecin $\mathrm{NH}_{2}$ analogue with Pseudomonas aeruginosa lipase

Table.1: Estimated free energy of binding (FEB) of cefovecin and its analogues against Pseudomonas aeruginosa lipase

\begin{tabular}{|c|c|c|}
\hline Substituent's & Structure & $\begin{array}{ll}\text { Docking } & \text { score } \\
(\mathrm{Kcal} / \mathrm{mol}) & \end{array}$ \\
\hline $\begin{array}{l}\mathrm{COOH} \\
\text { (Ceforecin) }\end{array}$ & $\mathrm{H}_{2}, \infty$ & -44.30 \\
\hline $\mathrm{COCH}_{3}$ & & -43.55 \\
\hline $\mathrm{CH}_{3}$ & & -39.49 \\
\hline $\mathrm{NO}_{2}$ & & -43.40 \\
\hline $\mathrm{CF}_{3}$ & & -30.25 \\
\hline $\mathrm{NH}_{2}$ & & -44.18 \\
\hline
\end{tabular}


To Estimated free energy of binding (FEB) of cefovecin and its analogues is shown in Table 1. Crystal structure of Pseudomonas aeruginosa lipase is shown in Figure 2. Cefovecin in complex with Pseudomonas aeruginosa lipase is shown in Figure 3 while the docked cefovecin analogues with Pseudomonas aeruginosa lipase are presented in Figures $4-8$. The Hydrogen bonding, electrostatic and steric interactions are depicted in Figures $9-14$. The binding energy of Cefovecin was -44.30 $\mathrm{kcal} / \mathrm{mol}$. The free binding energies of $\mathrm{COCH}_{3}, \mathrm{CH}_{3}, \mathrm{NO}_{2}$, $\mathrm{CF}_{3}$ and $\mathrm{NH}_{2}$ analogues were $-43.55,-39.49,-43.40$, 30.25 and $-44.18 \mathrm{Kcal} / \mathrm{mol}$ respectively. All the monosubstituted analogues showed lower negative values than the non substituted cefovecin. These lower negative values indicate that their inhibition is lower compared to than cefovecin against Pseudomonas aeruginosa lipase. The modes of bonding of six analogous structurally diverse cefovecin with Pseudomonas aeruginosa lipase were attributed to hydrogen bonding and steric interactions. Cefovecin and its monosubstituted analogues bonded to Pseudomonas aeruginosa lipase through Ser 211, Phe 19, Ile 142, Leu 138, Phe 214, Pro 210, Leu 118, Thr 114, Ser 218, Ala 217, Thr 221, and Phe 117.

\section{CONCLUSION}

The in silico binding energy of cefovecin and its analogues with Pseudomonas aeruginosa lipase showed that the reactions are feasible because of their negative values of free energy. The ability of these drugs to inhibit Pseudomonas aeruginosa lipase is assured. We therefore recommend the synthesis, characterization and clinical studies of these cefovecin derivatives.

\section{REFERENCES}

[1] Pfizer Animal Health. "Pfizer Convenia". Pfizer. Retrieved 29 January 2013.

[2] "European Public Assessment Report for Convenia (from the EMEA website)". Retrieved 2008-07-11.

[3] "FDA Approves First and Only Single-dose Antibiotic for Dogs and Cats" (Press release). Pfizer Inc. 2008-06-30. Retrieved 2008-07-11.

[4] Pfizer Animal Health. "Convenia". Pfizer. Retrieved 29 January 2013.

[5] Pfizer Animal Health. "Pfizer Convenia"(PDF). Pfizer. Retrieved 29 January 2013.

[6] Convenia Prescribing Information. "Convenia Prescribing Information". Pfizer. Retrieved 18 October 2015.

[7] Pfizer Animal Health Fact Sheet. "Pfizer Convenia". Pfizer. https://animalhealth.pfizer.com 8/11/2011
[8] Molegro Molecular Viewer, Molegro - a CLC bio company Finlandsgade 10-12 8200 Aarhus N Denmark http://www.clcbio.com, 2012

[9] Advanced Chemistry Development,http://www.acdlabs.com, 2008

[10] M.A.Thompson, ArgusLab 4.0, Planaria Software LLC, Seattle, WA. http://www.arguslab.com, 2007

[11] D. Duhovny, R. Nussinov, H.J. Wolfson, "Efficient Unbound Docking of Rigid Molecules', In Gusfield et al., Ed. Proceedings of the 2'nd Workshop on Algorithms in Bioinformatics (WABI), Springer Verlag, Rome, Italy, Lecture Notes in Computer Science , 2452, 185 - 200. 2002

[12] E. Mashiach, D. Schneidman-Duhovny, N. Andrusier, R. Nussinov, H.J. Wolfson, FireDock a webserver for fast interaction refinement in molecular docking, Nucleic Acid Res, vol. 36, pp. $229-292,2008$

[13] N. Andrusier, R. Nussinov, H.J. Wolfson. FireDock: fast interaction refinement in molecular docking proteins, vol. 69(1), pp.139 - 159, 2007

[14] B. Jayaram, T. Singh, G. Mukherjee, A. Mathur, S. Shekhar and V. Shekhar, "Sanjeevini: a freely accessible web-server for target directed lead molecule discovery", BMC Bioinformatics, vol. 13, pp. 7, 2012, http://www.biomedcentral.com/14712105/13/S17/S 7

[15] C.A. Lipinski CA, "Lead- and drug-like compounds: the rule-of-five revolution". Drug Discovery Today: Technologies, vol.1(4), pp.337-341, 2004 